\title{
Link-level Measurements of Outdoor 802.11g Links
}

\author{
Pulkit Gupta, Bharat Jain, Bhaskaran Raman, Purushottam Kulkarni \\ Department of Computer Science and Engineering \\ Indian Institute of Technology, Bombay, Mumbai 400076, India
}

\begin{abstract}
: 802.11 based outdoor community mesh networks have seen several deployments in the last few years. Prior linklevel studies focus mostly on $802.11 \mathrm{~b}$ links, and a systematic and detailed link-level study of $802.11 \mathrm{~g}$ is lacking. In this paper, we present a study of the link error rate as a function of the RSSI; we closely examine the RSSI threshold beyond which the link error rate is negligible. Our study is based on a wireless network testbed established in IIT Bombay. The testbed has nine nodes with links varying from $90 \mathrm{~m}$ to $1.2 \mathrm{~km}$. We find that the link abstraction holds in the case of $802.11 \mathrm{~g}$ and RSSI threshold range can be used for the predictable performance for such links. Significantly, we also observe a shift in the RSSI threshold for outdoor links, as compared to controlled experiments. We report the features of this shift, and analyze the possible causes. Additionally, we have measured the UDP saturation throughput of these links using two different platforms: Soekris net4521, and MikroTik RB433AH. We also document some of the significant unexpected experiences while conducting experiments on our testbed.
\end{abstract}

\section{INTRODUCTION}

WiFi-based mesh networks have seen tremendous growth over the last several years. For optimized performance, a detailed understanding of these networks is a must. Prior work has characterized medium distance (upto few hundreds of metres) [1], [17] as well as long-distance links [2]; these have considered $802.11 \mathrm{~b}$ links. In contrast, $802.11 \mathrm{a} / \mathrm{g} / \mathrm{n}$ variants of the technology have not seen such detailed and systematic study at the link level. While there have been some studies of $802.11 \mathrm{~g}$ mesh network links [13], [8], these have not focused on link-level performance measurements. In this paper, we complement prior measurement studies by undertaking a detailed link-level study of outdoor 802.11g links.

To carry out our experiments, we have established a wireless mesh network testbed in the IIT Bombay campus. The testbed has nine nodes, deployed on top of various buildings; most links have line-of-sight. The links were setup using $8 \mathrm{dBi}$ omni-directional antenna and link length between $90 \mathrm{~m}$ and $1.2 \mathrm{~km}$. We carried out our experiments on ten links. Figure 1 shows our testbed; the links in bold are the ones used for our measurement study. We have used Ubiquiti SR2 minipci wireless cards and performed our experiments using two different wireless platforms - Soekris (net4521) and MikroTik (RB433AH). Although our study consists of only ten links, the consistency of the results across the links indicates that our conclusions are indeed meaningful and can be considered as representative of $802.11 \mathrm{~g}$ based mesh network links.

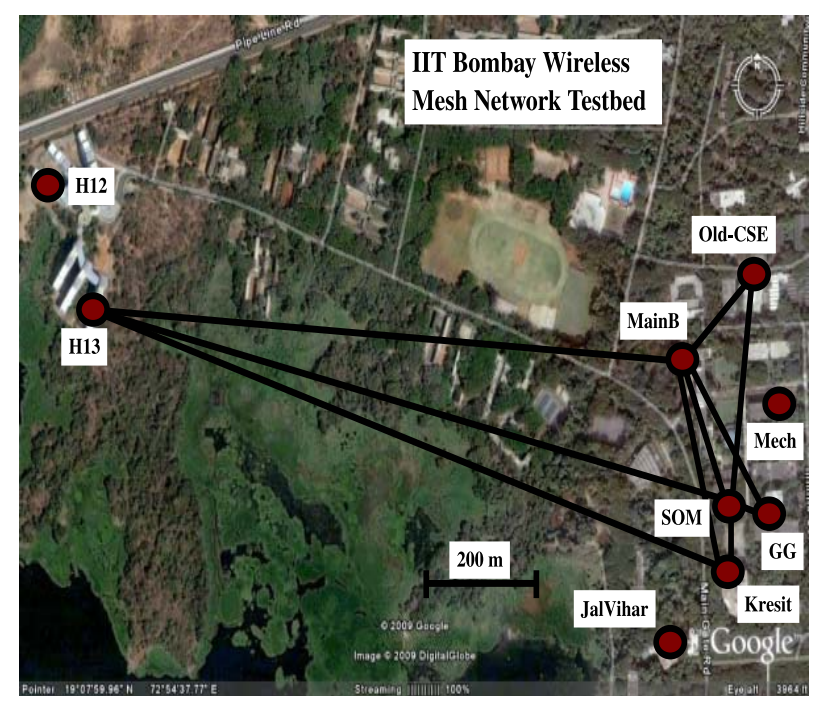

Fig. 1. IIT Bombay Wireless Mesh Network Testbed

It is known that link abstraction holds for $802.11 \mathrm{~b}$ links [2], [17]. This means that there exist a small window of Received Signal Strength Indication (RSSI) ${ }^{1}$ in which the packet error rate varies from almost negligible to $100 \%$. 802.11g uses Orthogonal Frequency-Division Multiplexing (OFDM) based modulation schemes, which is different from $802.11 \mathrm{~b}$; hence we seek to see if link abstraction holds for $802.11 \mathrm{~g}$ links as well.

In our prior work in [17], we have reported that link abstraction holds for $802.11 \mathrm{~g}$ links too. We confirm this with further measurements in this paper. In addition, this paper explores in detail the issue of what exactly is the RSSI threshold, for each $802.11 \mathrm{~g}$ data rate, beyond which link error rate becomes "small". We compare this threshold value as obtained from outdoor links with that obtained from controlled settings. We find that there is a significant shift in the threshold for outdoor links, as compared to controlled settings. The shift can be as high as 10-15 dB. In comparison, for the measurements reported in [17], the $802.11 \mathrm{~b}$ links show only a shift of 1$2 \mathrm{~dB}$ ([17] does not report the shift for 802.11g links).

Further, we observe that different outdoor links have different shifts. We also observe from our results that links which have a large shift for a particular transmit rate, also have a large shift for other transmit rates. We conjecture that such behavior is due to the differential attenuation of the $802.11 \mathrm{~g}$ sub-carriers and/or due to small-scale fading (within a packet

\footnotetext{
${ }^{1}$ Power of a radio signal measured at the receiver.
} 
duration, or within a few packets' duration).

In this paper, we also measure the UDP saturation throughput for each $802.11 \mathrm{~g}$ data rate and compare it with the theoretically achievable throughput. Our results indicate that the MikroTik platform is able to achieve the theoretically expected saturation throughput, while the slower Soekris platform is unable to do so ([2] reports a similar CPU bottleneck for 802.11 b, although on a different wireless card, without DMA support). This paper also documents some of our non-obvious experiences while performing experiments on the testbed.

The rest of the paper is organized as follows. The next section (Sec. II) talks about the related work. Sec III describes our experimental setup. Subsequently, Sec. IV presents an indepth analysis of the packet error rate and its dependence on RSSI. We then analyze the application throughput for $802.11 \mathrm{~g}$ rates in Sec. V. Sec. VI presents a few salient experiences during our experiments, and Sec. VII concludes the paper.

\section{RELATED WORK}

802.11b studies: The work in [1] represents an early measurement study of outdoor wireless mesh networks. [1] reports link-level measurements for medium length (a few hundred metres to a $\mathrm{km}$ or so) $802.11 \mathrm{~b}$ links in an urban setting. Our prior work in [17] presents similar measurements in campus and rural settings. The work in [2] presented detailed measurements of $802.11 \mathrm{~b}$ links in long-distance (a few $\mathrm{km}$ to several tens of $\mathrm{km}$ ) settings, in a rural region. These studies present various useful results related to the presence/absence of the link abstraction. In [5], the authors investigate commonly cited sources of packet loss induced by the wireless channel and by the $802.11 \mathrm{~b}$ MAC protocol. Client coverage area and throughput issues have also been measured in a semi-urban setting in [12]. A common feature of the above studies is that they have focused on $802.11 \mathrm{~b}$. In this paper, we seek to complement these efforts with a link-level study of $802.11 \mathrm{~g}$ mesh network links.

802.11g studies: Prior work on $802.11 \mathrm{~g}$ based wireless mesh networks includes: [13], [14], [15], [16], [8]. [13] reports measurements for long-distance $802.11 \mathrm{~g}$ links with directional antennas, and investigated the effects of interference between collocated directional antennas. Their measurements show that antenna orientation and placement along with channel separation at such a multi-hop node have a significant impact on the achievable throughput. The aim of the [8] was to evaluate the performance of long-distance $802.11 \mathrm{~g}$ links and study the impact of link-length on signal strength, average noise variation, free space path loss, jitter, and round-trip time.

[14] studies the effect of interference on 802.11g links; they study two simultaneous flows operating in non-overlapping channels. [16] provides extensive measurements on the comparative performance of $802.11 \mathrm{~b}$ and $802.11 \mathrm{~g}$ (for two rates, $6 \mathrm{Mbps}$ and $12 \mathrm{Mbps}$ ), and concluded that $802.11 \mathrm{~g}$ performs poorly compared to $802.11 \mathrm{~b}$ in an outdoor scenario, for reasons attributed to PHY errors, namely short PLCP preamble and limited tolerance of the cyclic prefix.
Our prior work in [17] presented measurements on 802.11g mesh links to show that the link abstraction holds.

In this paper, we complement the above prior studies in 802.11g. Our work focusses on an in-depth evaluation of the dependence of the link error rate on the RSSI. We study the RSSI threshold beyond which error rate drops to insignificant values. We compare this threshold across links, as well as with the threshold values computed using controlled experiments.

We also study the UDP saturation throughput on the mesh network links. While [3] too presents UDP and TCP throughput measurements, their focus was on finding the dependence on the size of packets, round trip time etc. We study the throughput as a function of the RSSI and report the presence/absence of other system bottlenecks in two different platforms.

\section{Measurement Setup And Methodology}

\section{A. Wireless Mesh Network Testbed Establishment}

Our wireless mesh network testbed consists of nine nodes distributed over roughly three square kilometers of the Indian Institute of Technology Bombay campus. Each node is deployed using water-proof boxes on the terrace of various buildings. We used $8 \mathrm{dBi}$ omnidirectional antenna (www.twinantennas.com) at both ends of the wireless mesh network link to achieve necessary transmit/receive gains for link setup. The set of site locations along with the links are shown in Figure 1. Table I shows link-length of the ten links used for the experiments.

TABLE I

SET OF LINKS USED FOR THE EXPERIMENTS

\begin{tabular}{|c|c|c|}
\hline End-point: 1 & End-point:2 & Length(m) \\
\hline \hline Kresit & SOM & 110 \\
Kresit & MainB & 320 \\
Kresit & H-13 & 1190 \\
SOM & MainB & 220 \\
SOM & GG & 90 \\
SOM & Old-CSE & 280 \\
SOM & H-13 & 1127 \\
MainB & GG & 250 \\
MainB & Old-CSE & 150 \\
MainB & H-13 & 1046 \\
\hline
\end{tabular}

\section{B. Hardware and Software Setup}

For most of our experiments, we used the Ubiquiti based SR2 cards (www.ubnt.com) as the WiFi radio. This card has two external connectors; the card is connected to an antenna using a pigtail and an LMR 200 RF cable. We performed experiments with two different platforms. First, we used the Soekris net4521 (www.soekris.com) platform to insert and use these cards. This $133 \mathrm{MHz}$ platform is popular in several outdoor community networks. Second, we used the $680 \mathrm{MHz}$ MikroTik-RB433AH platform (www.mikrotik.com).

We used the open-source Madwifi-trunk-3941 driver. We modified the driver to: (1) set the transmit power in steps of $1 \mathrm{dBm}$, and (2) to learn per-packet information such as the RSSI, silence level, MAC header details, etc.

We performed the same set of experiments in controlled settings too. For this, we took a Soekris (or MikroTik) board 
at both sender as well as the receiver side, connected them via an RF cable and various combinations of fixed step attenuators (3 dBm, $6 \mathrm{dBm}, 10 \mathrm{dBm}, 20 \mathrm{dBm}, 40 \mathrm{dBm}$ ) in-between.

\section{Measurement Methodology}

We consider the parameters (tx-power, tx-rate) that can affect the link characteristics and we perform experiments on the exhaustive set of these parameters. We perform experiments over the whole range of txpower $(0 \mathrm{dBm}-26 \mathrm{dBm})$ and for each rate of $802.11 \mathrm{~b}$ and $\mathrm{g}$ (1Mbps, $2 \mathrm{Mbps}, 5.5 \mathrm{Mbps}$, $6 \mathrm{Mbps}, 9 \mathrm{Mbps}$, 11Mbps, 12Mbps, 18Mbps, 24Mbps, 36Mbps, $48 \mathrm{Mbps}$, and 54Mbps). We chose a packet size of 1400 bytes (UDP payload), and a packet inter-arrival duration of $20 \mathrm{~ms}$ for our packet-error-rate experiments. We used $10(2.457 \mathrm{GHz})$ as the channel of operation and ahdemo mode for all nodes for all the experiments.

There are two broad metrics we consider during our measurements: (a) packet error-rate, and (b) UDP throughput ${ }^{2}$.

For each experiment, we choose a specific value for the transmit power, transmit rate and packet size. Within an experiment, we ran the experiment for a duration of $20 \mathrm{sec}$; this gives 1000 packet transmissions for the chosen inter-packet gap of $20 \mathrm{~ms}$. All the experiments were carried out by sending the MAC level broadcast packets, to prevent the effect of MAC level ACKs.

For the packet error-rate experiments, in order to get the range of RSSI between $-60 \mathrm{dBm}$ and $-95 \mathrm{dBm}$, we had to repeat our whole set of packet error-rate experiments using $10 \mathrm{dBm}$ and $20 \mathrm{dBm}$ external RF attenuators.

\section{Analysis of Packet Error-Rate}

\section{A. RSSI threshold range for $802.11 \mathrm{~g}$}

In order to study the correlation between error rate of transmitted packets and the received signal strength at receiver, we varied txpower in combination with the different attenuator values. Table II shows the RSSI values of 10 percent and 90 percent packet error rate for ten outdoor links and two controlled experiments for each rate. We have also shown the packet error rate for each rate of $802.11 \mathrm{~b}$ (1Mbps, 2Mbps, 5.5Mbps and $11 \mathrm{Mbps}$ ).

In case of controlled experiments, the RSSI band is $3-4 \mathrm{dBm}$ for the error rate to change from 10 percent to 90 percent. On the other hand, the band for most of the outdoor links is 5$10 \mathrm{dBm}$. Blank entries indicate experiments in which we could not obtain the RSSI band corresponding to the 10 percent to 90 percent error rate over the feasible range of RSSI values we could generate.

Detailed distribution of error rate as a function of RSSI for five outdoor links and a controlled experiment for transmit rates of $2 \mathrm{Mbps}, 6 \mathrm{Mbps}, 18 \mathrm{Mbps}$ and $48 \mathrm{Mbps}$ are shown in Figures 2, 3, 4 and 5 respectively. The range of RSSI values for which the error rate decreases from $90 \%$ to $10 \%$, and beyond which the error rate is considered low,

\footnotetext{
${ }^{2}$ We do not consider TCP throughput since we do not expect any MAC level ACK timeouts in these short/medium length links, unlike in [2].
}

is the RSSI threshold range. As seen from the figures, the threshold for higher transmit rates is higher, e.g., considering the MainB_GG link, the RSSI threshold changes from $92 \mathrm{dBm}--84 \mathrm{dBm}$ for a transmit rate of $1 \mathrm{Mbps}$, to $-83 \mathrm{dBm}-$ $-81 \mathrm{dBm}$ for $6 \mathrm{Mbps}$, to $-85 \mathrm{dBm}--76 \mathrm{dBm}$ for $18 \mathrm{Mbps}$ and $-75 \mathrm{dBm}--68 \mathrm{dBm}$ for 54 Mbps.

Our main observations based on the controlled-indoor and outdoor experiments are as follows:

- For each transmit rate of $802.11 \mathrm{~g}$, there is a narrow RSSI threshold range where packet error-rate decrease from 100 percent to 0 percent. However, as compared to these results, we observed a shift in the RSSI threshold for all the outdoor links. The shift is visible for $802.11 \mathrm{~b}$ as well and the trend looks similar for $802.11 \mathrm{~b}$ rates as well. Although [17] analyzed the RSSI threshold range for $802.11 \mathrm{~b}$ rates, they did not observe the shift in RSSI threshold for different links which is clearly visible on their results.

- Among the outdoor links, the one which has higher shift for one rate, has higher shift across all the rates. For example, as seen in Figures 2, 3, 4 and 5, the RSSI threshold range for $2 \mathrm{Mbps}, 6 \mathrm{Mbps}, 18 \mathrm{Mbps}$ and $48 \mathrm{Mbps}$ follows a pattern of Control_Expt, MainB_CSE, SOM_H-13 and MainB_GG, MainB_SOM, Kresit_MainB from left to right. It means that the shift is consistent across the various links. In our prior work in [17], we did similar measurements for some rates of $802.11 \mathrm{~g}$ one and half years ago but we did not note this behavior at that time. However, one can see that the same observation holds in the graphs in [17] too. We also found the similar behavior during our previous measurement studies [18].

\section{B. Analysis of Shift in RSSI}

Ideally, we expect to get the same RSSI threshold range for controlled experiments as well as outdoor links. However, for almost all the links, we observe a shift of around $10-15 \mathrm{~dB}$ across each transmit rate for most of the links. For comparison, we also performed experiments using a $2.4 \mathrm{GHz} 802.15 .4$ platform. We used the Tmote platform (www.moteiv.com). Surprisingly, we did not observe any shift in the RSSI threshold (in controlled setting as well as on our testbed links, the RSSI threshold was $-90 \mathrm{dBm}$ to $-92 \mathrm{dBm}$ ). So the shift observed cannot be due to the environment alone. We tried to explore different potential reasons of this shift.

Position of Antenna: We repeated RSSI experiments on the two links (MainB_OldCSE and MainB_SOM) by changing the vertical position of the antenna by $1 \mathrm{ft}$. Even after changing the vertical position of the antenna, there was no difference in the RSSI threshold across all the rates of $802.11 \mathrm{~g}$.

Hardware Issues: Since we were getting shifts in the outdoor links from the indoor settings, we suspect some strange behavior from the hardware. We repeated the experiments by swapping the hardware (MikroTik boards, pigtails and network cards) between indoor experiments and one of the outdoor links (MainB_SOM). We observed that the RSSI threshold 


\begin{tabular}{|c|c|c|c|c|c|c|c|c|c|c|c|c|c|c|c|c|c|c|c|c|c|c|c|c|}
\hline Modulation(802.11b/g) & \multicolumn{2}{|c|}{ b } & \multirow{2}{*}{\multicolumn{2}{|c|}{ b }} & \multirow{2}{*}{\multicolumn{2}{|c|}{$\frac{b}{55}$}} & \multirow{2}{*}{\multicolumn{2}{|c|}{$\mathrm{b}$}} & \multirow{2}{*}{\multicolumn{2}{|c|}{$\mathbf{g}$}} & \multicolumn{2}{|c|}{$\mathrm{g}$} & \multicolumn{2}{|c|}{$\mathrm{g}$} & \multicolumn{2}{|c|}{ g } & \multicolumn{2}{|c|}{ 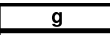 } & \multicolumn{2}{|c|}{$\mathrm{g}$} & \multicolumn{2}{|c|}{$\mathrm{g}$} & \multicolumn{2}{|c|}{$\mathrm{g}$} \\
\hline Link \Rate (Mbps) & & 1 & & & & & 1 & & & & 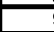 & 9 & & 2 & 1 & & 2 & & 3 & & 4 & & 5 & \\
\hline (percentage error) & 10 & 90 & 10 & 90 & 10 & 90 & 10 & 90 & 10 & 90 & 10 & 90 & 10 & 90 & 10 & 90 & 10 & 90 & 10 & 90 & 10 & 90 & 10 & 90 \\
\hline Control_Expt_MikroTik & & & -92 & -94 & -90.6 & -99.4 & -88.8 & -92 & & & & & -90.8 & -94.4 & & & -82.8 & -86 & -80.2 & -83.2 & -77.4 & -83.5 & -76.5 & -79.1 \\
\hline Control_Expt_Soekris & & & -81.7 & -95 & -92 & -94.3 & & & -90 & $\mid-93.3$ & & & & & -86.4 & -91.7 & & & -80 & -82 & & & & \\
\hline Kresit_H13 & -83 & -89 & -79 & $\mid-85.1$ & -80 & -90.4 & -76.5 & -87.3 & -78.6 & -90 & -75.4 & -88.1 & -70.4 & -90.8 & -70 & -87 & -73 & -84.1 & & & & & & \\
\hline Kresit_MainB_Soekris & -85 & & -81 & -86 & -85 & & & & -72 & -80 & -71 & -78 & & & -71 & -77 & -70 & -78 & & & & & & \\
\hline Kresit_SOM & -76 & $\mid-89.9$ & -79.6 & -85.5 & $\mid-74$ & -82 & -78 & -81.2 & -75 & -85.3 & -74.5 & -84.1 & -70 & -83.7 & -71 & -82.8 & -68 & -85.1 & & & & & & \\
\hline MainB_OldCSE & -89 & -93 & -83 & $|-93.1|$ & & & & & & & -80 & -92 & & & & & -82 & -86.7 & -80 & -89.6 & -71 & -79 & -71.9 & -78.3 \\
\hline MainB_GG & -84 & -92 & -81.5 & $|-87.1|$ & -83.3 & -87.7 & -76.7 & -87.1 & -81 & -83 & -76 & -83.5 & -78 & -84.3 & -76 & -85.1 & -76 & -81.5 & -69.3 & -82.5 & -68.2 & -78 & -68.1 & -75 \\
\hline MainB_H13 & -82 & $|-93.7|$ & -81.7 & -86 & -81 & -86 & -79.1 & -84.1 & -79 & -88 & -77.4 & -85 & -77.6 & -86 & -77.5 & -85.1 & & & & & & & & \\
\hline MainB_SOM & -80 & -84 & -76 & -83 & -77 & -87 & $\mid-77.8$ & -87.1 & -75 & -83.6 & -76 & -83 & -77 & -83 & -72 & -83 & -70.7 & -81.3 & -65 & \begin{tabular}{|l|}
-79 \\
\end{tabular} & -66.1 & -77 & -64.9 & $\mid-73.8$ \\
\hline SOM_OldCSE & -88.7 & $|-96.8|$ & -87.5 & -92 & -87.3 & -94 & & & -80.4 & $\mid-90.4$ & -83 & -89.1 & -79.8 & -89.2 & & & & & & & & & & \\
\hline SOM_GG & -84 & $|-92.1|$ & -80 & $|-87.1|$ & $\mid-79.9$ & -87 & -81 & -90 & -78 & -83 & -76 & -83 & -79 & -83.7 & -76 & $\mid-82.2$ & $\mid-74.1$ & -83 & -63.3 & -82 & & & & \\
\hline SOM_H13 & -82 & -89.6 & -79 & -85.3 & -79 & -87 & -76.9 & -86.4 & -80 & -92 & -76 & -88 & -77 & -89 & -74 & -86 & -69.1 & -83.9 & -71.6 & -79.1 & & & & \\
\hline
\end{tabular}

TABLE II

RSSI THRESHOLD RANGE FOR 802.11B AND 802.11G

values for both setups was similar, with similar shift with the outdoor link.

External Interference: Interference, one of the primary reasons for poor links is another possible reason for the shift. We have performed experiments on different links several times, across many days and also at different times. For each experiment, over all the transmit rates, we observed that all the outdoor links show a RSSI threshold shift of 10-15 dB. Since interference effects are mostly transient and probability that during all our experiments there was a steady source of interference is low, it is unlikely that interference is a reason for the RSSI shift. While this is our conjecture based on our experiments so far, we are pursuing this direction further to determine the possible role of interference in effecting the RSSI threshold shift.

Differential attenuation of OFDM sub-carriers: It is likely that the different sub-carrier frequencies of the OFDM modulation used face different levels of attenuation, but the card reports only an aggregate value. In such a case, if the RSSI threshold of one of the sub-carriers goes down, the aggregate RSSI reported does not change much, but packet error rate is affected anyway since the bit-stream encoded using the lowRSSI sub-carrier is affected. This could explain the observed shift in the RSSI plot. However, we observed such shift for the 802.11 b rates too, in the links, which do not use OFDM modulation.

Fast fading: If we have fast fading within a packet, or within a small collection of packets, then we could have a case where the estimated RSSI is an overestimate. This is because we are able to compute the RSSI of only the received packets and not the packets which were lost. This could also result in the observed shift in the RSSI graph. However, we wish to note that we observed much less RSSI variability (fading) in our experiments with the 802.15.4 platform, as compared to the 802.11 platform $(0.01 \mathrm{~dB}$ standard deviation versus $>1 \mathrm{~dB}$, respectively).

In summary, we suspect that either the differential attenuation of the OFDM sub-carriers and/or fast fading is causing the observed shift. We are in the process of designing appropriate experiments to examine this further.

Implications of RSSI Threshold: Link abstraction refers to whether one can deterministically consider a link to exist or not. Similar to $802.11 \mathrm{~b}$ [17], from our experiments we conclude that link abstraction holds in the case of $802.11 \mathrm{~g}$ as well. All of our links exhibit a RSSI threshold band of 5-10 dB on either side of which the packet error rate is $0 \%$ and $100 \%$. Thus, for all RSSI values that lie beyond this range, we can deterministically conclude the existence (or non-existence) of the link. However, possibly due to reasons of interference and propagation effects with the OFDM modulation scheme the RSSI threshold is shifted; this is something not noted in [17].

\section{UDP THROUGHPUT ANALYSIS}

In packet error-rate experiments, we found that for each rate of $802.11 \mathrm{~g}$, there exists a definite value of RSSI beyond which the packet error-rate is close to zero. If we operate in the region above the RSSI threshold, we expect to get the saturation throughput. The main goal of the experiments in this section is to verify whether we can achieve theoretical UDP saturation throughput on outdoor links. We measured and analyzed the UDP saturation throughput on our testbed links using both the Soekris and MikroTik platforms.

We calculated the theoretical UDP saturation throughput as follows, A packet transmission includes several factors, the DIFS, time to transmit the synchronization header, data transmission time and signal extension time. All the above factors except data transmission time have a fixed time duration. Table III shows the typical values of these parameters.

TABLE III

802.11G STANDARD VALUES

\begin{tabular}{|c|c|}
\hline Factors & Time $(\mu$ sec. $)$ \\
\hline DIFS & 28 \\
Header for Synch. & 20 \\
Signal Extension & 6 \\
\hline
\end{tabular}

The time for sending the data depends on the number of data bits per symbol. The symbol lengths for $6 \mathrm{Mbps}, 9 \mathrm{Mbps}$, $12 \mathrm{Mbps}, 18 \mathrm{Mbps}, 24 \mathrm{Mbps}, 36 \mathrm{Mbps}$, 48Mbps and 54Mbps are 24, 36, 48, 72, 96, 144, 192 and 216 bits respectively and duration of each symbol is $4 \mu \mathrm{sec}$. For a payload of 1400 bytes, the total time for transmission at $54 \mathrm{Mbps}$ is, $28 \mu \mathrm{s}+20 \mu \mathrm{s}+$ $(52 \times 4 \mu \mathrm{s})+6 \mu \mathrm{s}=264 \mu \mathrm{s}$, resulting in a throughput of 40.76 Mbps above the MAC layer. The theoretical UDP saturation throughput values for each rate of $802.11 \mathrm{~b} / \mathrm{g}$ are reported in Table IV. 


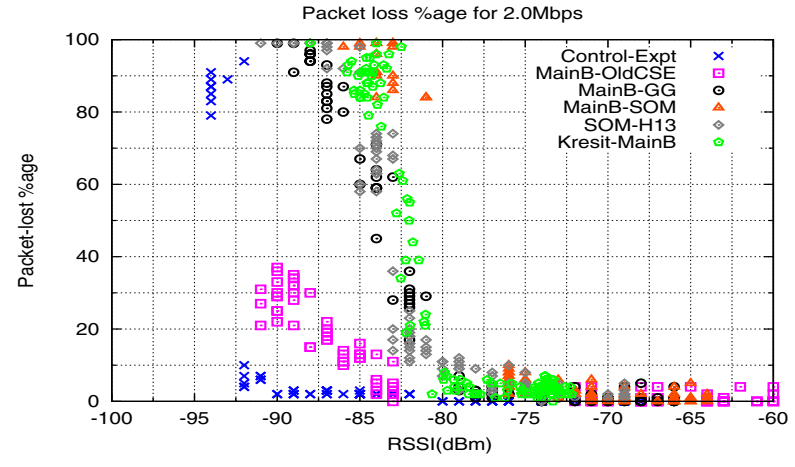

Fig. 2. RSSI Threshold for $802.1 \mathrm{~b}-2 \mathrm{Mbps}$

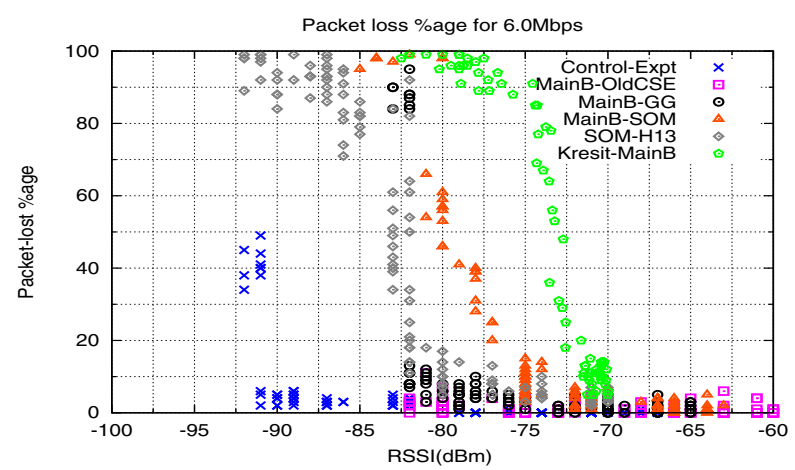

Fig. 3. RSSI Threshold for $802.11 \mathrm{~g}-6 \mathrm{Mbps}$

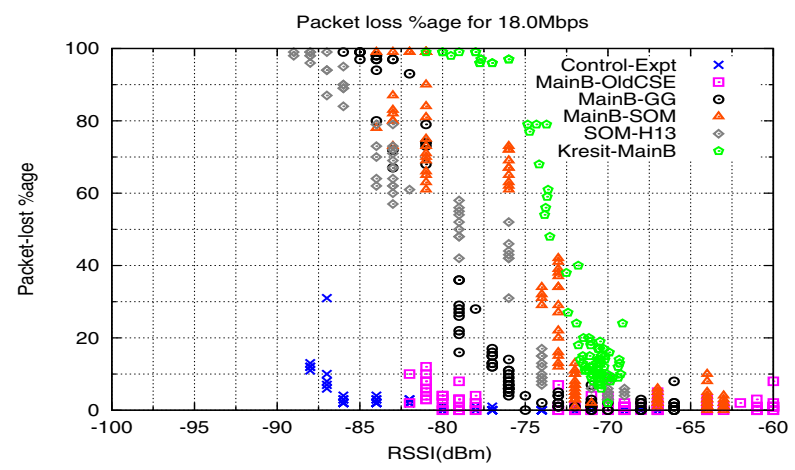

Fig. 4. RSSI Threshold for $802.11 \mathrm{~g}-18 \mathrm{Mbps}$

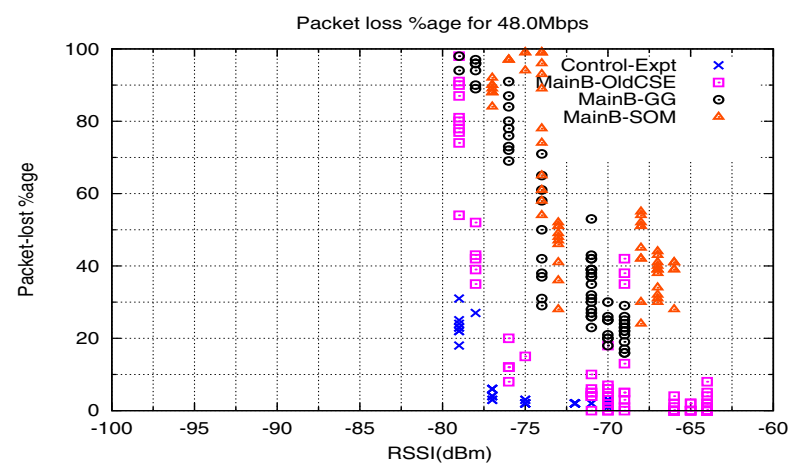

Fig. 5. RSSI Threshold for $802.11 \mathrm{~g}-48 \mathrm{Mbps}$

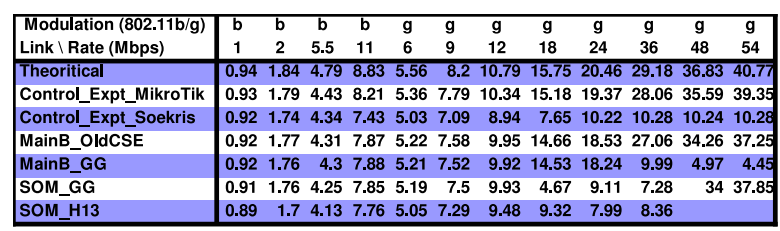

TABLE IV

UDP SATURATION THROUGHPUT FOR 802.11G

We perform UDP experiments on the links by running a socket sender/receiver application at the two ends for 40 seconds with a packet size of 1400bytes. We use two different platforms, i.e., Soekris and MikroTik to verify whether is there any effect of hardware on the UDP saturation throughput. We expect the UDP saturation throughput to achieve its theoretical value on the outdoor links as well.

Table IV shows UDP throughput obtained in controlledindoor and outdoor settings for the different transmit rates and the theoretical expected throughput. With the MikroTik platform, at all transmit rates, in a controlled-indoor setting, the UDP throughput is close to the theoretical maximum.

With the Soekris platform, the controlled experiments yielded different results. For transmit rates of $1 \mathrm{Mbps}, 2 \mathrm{Mbps}$ and $5.5 \mathrm{Mbps}$, we found the UDP throughput values to be quite comparable with theoretical values. However, beyond $5.5 \mathrm{Mbps}$, the difference between the experimentally achieved and the theoretical value starts increasing, the difference increasing further for higher rates. For transmit rate of $54 \mathrm{Mbps}$, the maximum UDP throughput using the Soekris platform was $10.29 \mathrm{Mbps}$ as compared to the theoretical value of 39.328 Mbps. [2] reported the same behavior for the $11 \mathrm{Mbps}$ transmit rate of $802.11 \mathrm{~b}$ with the Soekris platform, although with Prism2 cards, which did not support DMA. With our Ubiquiti cards too, the low throughput values in the case of Soekris may be attributed to the low processsing capability of Soekris $(133 \mathrm{MHz}$ for net 4521 and $266 \mathrm{MHz}$ for net4826) as compared to the MikroTik (680MHz).

Next, we measured the throughput on the outdoor links and observed different types of behavior. As seen in the Table IV, throughput on the MainB_OldCSE and $S O M_{-} G G$ links are similar to that reported on the controlled experiments with the MikroTik platform. The other links show increasing and near-theoretical throughput values for certain transmit rates, and significant deviation from the theoretical for others. For example, the MainB_GG link yields a throughput of 18.24 Mbps at a transmit rate of 24 Mbps, but only $25.5 \mathrm{Mbps}$ and 20.6 Mbps for transmit rates of $48 \mathrm{Mbps}$ and $54 \mathrm{Mbps}$ respectively. The reason for this behavior is that the links are operating close to the RSSI threshold in those cases.

Implications: If the RSSI at the receiver is more than the threshold for low error rate, the achievable UDP throughput is close (within 1-2 Mbps in the worst case) to that of the theoretical maximum. Applications like video which require high data rates, can use this result of expected achievable throughput in their design. Another point to note is that the 
achievable throughput depends on the hardware capabilities, the Soekris platform in our case.

\section{EXPERIENCES WITH SOEKRIS AND MIKROTIK}

We used a combination of Linux-2.6.25.4 and Madwifi-0.9.4 for performing our earlier experiments with Soekris net4521. However, during our experiments on the testbed links, we got some unexpected errors related with the Soekris. The flash file system used to get corrupted frequently. This problem surfaced in terms of error messages reporting "NFS stale" for certain directories. At other times, the file-system corruption wiped out the kernel and/or the grub boot image itself.

We encountered this problem in new Soekris boxes as well, so it could not have been due to bad flash sectors. Reinstallation of the kernel, and reformatting of the file system did not help either. Then by examining the $\log$ entries of syslog and dmesg, we suspected the reason to be something related with the drivers and kernel versions. So, we tried using different combinations of Madwifi versions (Madwifi-0.9.4, Madwifi-ng and Madwifi-trunk-3941) and other Linux kernel versions (Linux-2.6.25.4, Linux 2.6.26, Linux-2.6.27.2 and Linux-2.6.28.7). We also explored the other potential reasons behind these problems like improper implementation of our software, hardware problem or excessive writing to the flash but none of them turned out to be the main reason for all these problems. Finally, the frequency of these errors was decreased by using Madwifi-trunk-3941 and Linux-2.6.28.7 [18]. Given that several research efforts have used the Soekris platform for outdoor mesh links, it is quite surprising that no one has reported it whereas we encountered them consistently.

We explored a new platform, MikroTik, for establishing our testbed and found its performance to be better in terms of processing capability, booting time etc. In order to install kernel drivers on this platform, we used a firmware program called Openwrt[19] which is used for cross-compiling the drivers for particular architecture. Openwrt uses its application kamikaze where one can choose different set of platforms and drivers for making the .bin files for a target architecture. By default, Openwrt uses Linux-2.6.26.5 and Madwifi-0.9.3 for the installation for MikroTik architecture. We tried to use Madwifi-0.9.4 and Madwifi-ng for compilation using Openwrt, but could not succeed in that. The problem was that the Madwifi-0.9.4 and Madwifi-ng do not support the MikroTik hardware platform. Then we tried the latest version of Madwifi, Madwifi-trunk-3941 for this architecture and fortunately it worked. It is likely that in the latest version of Madwifi, support for MikroTik architecture is included. Overall, during our testbed experiments, we found the MikroTik boards to be quite reliable.

\section{CONCLUSION}

In this paper, we have presented a link-level measurement study of $802.11 \mathrm{~g}$ based outdoor wireless mesh network links. Our main conclusion is that while the link abstraction holds in case of $802.11 \mathrm{~g}$ as well, the RSSI threshold exhibits a shift from that observed in controlled settings. We conjecture that the differential propagation behavior of the sub-carriers of OFDM affects the RSSI threshold range considerably. Although we carried out our experiments only for ten links, consistency of the results across the links verifies that we can expect a similar behavior from a similar deployment using 802.11g wireless mesh network links.

We have also calculated the UDP saturation throughput and found that we can achieve the theoretical values in the absence of any hardware/software bottlenecks. To conclude, we believe that our measurements and the documentation of our experience will help future $802.11 \mathrm{~g}$ mesh network link diagnosis and planning.

Acknowledgment: This work was supported by the Tata Telecom Centre (IIT Bombay).

\section{REFERENCES}

[1] Daniel Aguayo, John Bicket, Sanjit Biswas, Glenn Judd, Robert Morris, "Link-level Measurements from an 802.11 b Mesh Network," in SIGCOMM, Aug 2004.

[2] Kameswari Chebrolu, Bhaskaran Raman, and Sayandeep Sen, "LongDistance 802.11b Links: Performance Measurements and Experience," in 12th Annual International Conference on Mobile Computing and Networking (MOBICOM), Sep 2006, Los Angeles, USA.

[3] Rabin Patra, Sergiu Nedevschi, Sonesh Surana, Anmol Sheth, Lakshminarayanan Subaramanian Eric Brewer, "WiLDNet: Design and Implementation of High Performance Wifi Based Long Distance Networks", USENIX NSDI, Apr 2007.

[4] Roger P. Karrer, Istvan Matyasovszki, Alessio Botta, Antonio Pescape, "Experimental Evaluation and Characterization of the Magnets Wireless Backbone," in WiNTECH, Sept 29, 2006.

[5] A. Sheth, S. Nedevschi, R. Patra, S. Surana, E. Brewer, and L. Subramanian, "Packet Loss Characterization in WiFi-based Long Distance Networks," in INFOCOM, May 2007.

[6] Wireless Community Network List, in http://www.toaster.net/wireless/community.html.

[7] Wray Community Communications, in http://www.wrayvillage.co.uk/wraycomcomhome.htm.

[8] Hesham El-Sayed, Sherali Zeadally, Mohamed Boulmalf, "Experimental Evaluation and Characterization of Long-distance 802.11g Links", ICN 2008.

[9] Pulkit Gupta, Seminar Report on "Wireless Measurements Studies", Dept of Computer Science, IIT Bombay, April 2008

[10] Pulkit Gupta, MTPStage-1 Report on "RSSI Based Rate Setting in Wireless Mesh Networks", Dept of Computer Science, IIT Bombay, July 2008

[11] http://witestlab.poly.edu/wiki/MadwifiTutorial.

[12] Joshua Robinson and Edward W. Knightly, "A Performance Study of Deployment Factors in Wireless Mesh Networks", INFOCOM 2007.

[13] Timothy Ireland, Adam Nyzio, Michael Zink, and Jim Kurose, "802.11g Long-distance Measurements: Antenna Placement and Orientation" in WinMee, April 2007

[14] Ashish Sharma, Ramya Raghavendra, Krishna Puttaswamy, Henrik Lundgren, Kevin Almeroth, Elizabeth Belding-Royer, "Experimental Characterization of Interference in a 802.11g Wireless Mesh Network", White Paper, http://www.cs.ucsb.edu/ asharma/ Interferencecharacterization.pdf

[15] Stefan Achleitner and Wolfgang Seiss, Master's thesis, "Performance Measurements in Wireless 802.11g Multi-Hop Networks", 2006.

[16] Giuseppe Bianchi, Fabrizio Formisano, Domenico Giustiniano, "802.11b/g Link Level Measurements for an Outdoor Wireless Campus Network", in WoWMoM, 2006

[17] Bhaskaran Raman, Kameswari Chebrolu, Dattatraya Gokhale, Sayandeep Sen, "On the Feasibility of the Link Abstraction in Wireless Mesh Networks", Accepted for publication, IEEE Transactions on Networking.

[18] Pulkit Gupta, MTPStage-2 Report on "RSSI Based Rate Setting in Wireless Mesh Networks", Dept of Computer Science, IIT Bombay, Dec 2008

[19] Openwrt Community, in http://www.openwrt.org 